\title{
Isolation of Besnoitia besnoiti from infected cattle in Portugal
}

\author{
$\mathrm{H}_{x}$ Cortes $^{\mathrm{a}, *}$, Y. Reis ${ }^{\mathrm{b}, \mathrm{c}}$, H. Waap ${ }^{\mathrm{b}}$, R. Vidal ${ }^{\mathrm{d}}$, H. Soares ${ }^{\mathrm{c}, \mathrm{e}}$, I. Marques ${ }^{\mathrm{e}}$, \\ I. Pereira da Fonseca ${ }^{\mathrm{f}}$, I. Fazendeiro ${ }^{\mathrm{f}}$, M.L. Ferreira ${ }^{\mathrm{f}}$, V. Caeiro ${ }^{\mathrm{a}}$, \\ V. Shkap ${ }^{\mathrm{g}}$, A. Hemphill ${ }^{\mathrm{h}}$, A. Leitão ${ }^{\mathrm{b}}$ \\ ${ }^{a}$ Laboratório de Parasitologia, ICAM, Núcleo da Mitra, Universidade de Évora, Ap 94, \\ Évora 7002-554, Portugal \\ ${ }^{\mathrm{b}}$ Instituto de Investigação Científica Tropical, CIISA, Lisboa, Portugal \\ ${ }^{\mathrm{c}}$ Instituto Gulbenkian de Ciência, Oeiras, Portugal \\ ${ }^{\mathrm{d}}$ Faculdade de Farmácia, Laboratório de Engenharia Genética, Universidade de Lisboa, Portugal \\ ${ }^{\mathrm{e}}$ Escola Superior de Tecnologia da Saúde de Lisboa, Portugal \\ ${ }^{\mathrm{f}}$ Faculdade de Medicina Veterinária de Lisboa (CIISA), Portugal \\ ${ }^{\mathrm{g}}$ Division of Parasitology, Kimron Veterinary Institute, Bet Dagan, Israel \\ ${ }^{\mathrm{h}}$ Institute of Parasitology, Vetsuisse Faculty, University of Bern, Bern, Switzerland
}

Received 9 November 2005; received in revised form 28 April 2006; accepted 11 May 2006

\begin{abstract}
Besnoitia besnoiti, an obligate intracellular protozoan parasite belonging to the phylum apicomplexa, and is the causative agent of bovine besnoitiosis. Besnoitiosis is responsible for significant losses in the cattle industry of Africa and Mediterranean countries due to the high morbidity rate, abortion and infertility in males. The acute stage of disease is associated with the proliferative forms (tachyzoites) and is characterized by fever, whimpery, general weakness and swelling of the superficial lymph nodes. During the following chronic stage, a huge number of cysts are formed mainly in the subcutaneous tissues. This process is non-reversible, and chronic besnoitiosis is characterized by hyper-sclerodermia, hyperkeratosis, alopecia and, in bulls, atrophy, sclerosis and focal necrosis that cause irreversible lesions in the testis.

In this paper we report on the identification of large cysts in the skin of a cow and a bull in Portugal, which presented loss of hair and enlargement and pachydermis all over the body. The observation of a two-layered cyst wall within the host cell, the encapsulation of the host cell by a large outer cyst wall, and the subcutaneous localization of the cysts within the host, were characteristic for $B$. besnoiti. The parasites were isolated from the infected animals and successfully propagated in Vero cells without prior passages in laboratory animals. Morphological characterization of $B$. besnoiti tachyzoites and the amplification of the $149 \mathrm{bp}$ segment from the internal transcribed spacer 1 (ITS1), aided with specific primers, confirmed the identification of $B$. besnoiti.
\end{abstract}

(C) 2006 Elsevier B.V. All rights reserved.

Keywords: Besnoitia besnoiti; Bovine besnoitiosis; Portugal; Cyst; In vitro cell culture

* Corresponding author. Tel.: +351 965017554; fax: +351268639667.

E-mail address: hcec@uevora.pt $\left(\mathrm{H}_{\kappa}\right.$ Cortes $)$.

\section{Introduction}

Besnoitia besnoiti, the causative agent of bovine besnoitiosis, is an obligate intracellular parasite, belonging to the Sarcocystidae family. This protozoan parasite was first described in France by Besnoit and 
Robin (1912). Three years later Franco and Borges (1915) published a study on the occurrence of bovine besnoitiosis in Portugal, based on animals rejected in a slaughterhouse between $1887_{\Lambda}$ and 1915. All these animals came from Alentejo, south of Portugal (Franco and Borges, 1915). Subsequently, the occurrence of the disease in Europe received little attention until the last decade of the 20th century when it was reported in Spain (Juste et al., 1990), Portugal (Cortes et al., 2003; Cortes et al., 2005) and France (Bourdeau et al., 2004).

A herd study on clinical cases of bovine besnoitiosis showed that the fatality rate is usually about 10\% (Pols, 1960) despite the fact that 70-90\% of animals had specific antibodies directed against $B$. besnoiti (Bigalke, 1968). Thus, most animals die during the chronic stage of infection. Only few studies have been carried out on the early stage mechanisms of $B$. besnoiti infection and immune pathways (Basson et al., 1970; Bigalke et al., 1974; Bigalke, 1967, 1968, 1960).

Due to the isolation of $B$. besnoiti from farm animals and the development of in vitro culture further investigations on the transmission and epidemiology of besnoitiosis (Bigalke, 1968; Janitschke et al., 1984) were possible. In addition, in vitro culture of $B$. besnoiti allowed the production of attenuated parasites, which were then used as an experimental vaccine in South Africa (Bigalke et al., 1974) and Israel (Pipano, 1997), and this has lead to the beginning of studies on the immunology (Shkap et al., 1989, 1990, 2002; Shkap and Pipano, 1993), chemotherapy (Elsheikha and Mansfield, 2004; Shkap et al., 1987b), phylogeny, and ultrastructure of Besnoitia (Dubey et al., 2003; Ellis et al., 2000; Shkap et al., 1988).

So far B. besnoiti has been isolated after several passages in laboratory animals, namely rabbits in South Africa (Pols, 1954) and gerbils in Israel (Meriones tristrami Shawii) (Neuman, 1974), prior to adaptation to in vitro culture. Subsequently, additional isolates were produced in South Africa (Bigalke, 1968) and in Israel (Shkap et al., 1987a) using the same approach. No isolates from other geographical areas have been reported to date.

The present paper describes two B. besnoiti isolates from Portugal. These isolates described in this report could be propagated under in vitro conditions without the need of laboratory animals for merozoite adaptation. These results will contribute to the research on this etiological agent of a potentially significant disease in cattle.

\section{Material and methods}

\subsection{Identification of cattle potentially infected with Besnoitia}

Subsequently to a case of bovine besnoitiosis identified by Malta and Silva in Portugal (1984, data not published), veterinarians were actively contacted in order to obtain material for further studies B. besnoiti. Cattle presenting chronic manifestations of skin disease were subjected to a skin biopsy and serum collection. Skin biopsies were performed using biopsy punch $(\varnothing 8 \mathrm{~mm})$, were fixed in $10 \%$ formalin, embedded in paraffin, and $3-5 \mu \mathrm{m}$ sections were processed for hematoxilin/eosin (H/E) staining. $B$. besnoiti tissue cysts were identified by light microscopy.

\subsection{Culture of Vero cells}

Vero cells (ATCC-CCL81) were cultured in T-25 tissue culture flasks, and were maintained in Dulbecco's modified eagle culture medium (D-MEM with $100 \mathrm{U}$ penicillin/ml, $\quad 100 \mu \mathrm{g} / \mathrm{streptomycin} / \mathrm{ml}$ and $0.25 \mu \mathrm{g}$ amphotericin $\mathrm{B} / \mathrm{ml}$ ) in a humidified incubator with $5 \% \mathrm{CO}_{2}$ atmosphere. Confluent monolayers were passaged routinely every 6 days.

\subsection{Isolation and tissue culture of B. besnoiti}

One 4 years old cow from Sallers' breed, and a 6 years old Charolais bull, from two separate farms in Évora region (south-east of Portugal), both exhibiting clinical features reminiscent for besnoitiosis were culled, and subcutaneous tissues from these animals were collected, stored at $4{ }^{\circ} \mathrm{C}$, and transported to the laboratory. With a scalpel, tissue pieces showing cysts of $B$. besnoiti were collected in a Petri dish containing PBS plus $100 \mathrm{U}$ penicillin $/ \mathrm{ml}, 100 \mu \mathrm{g}$ streptomycin $/ \mathrm{ml}$, and $0,25 \mu \mathrm{g}$ amphotericin $\mathrm{B} / \mathrm{ml}$, and were washed twice with this solution. The endozoites were freed from the large cysts by scattering the tissue with an $18 \mathrm{G}$ needle.

The PBS with liberated bradyzoites was collected and centrifuged at $770 \times g$ for $15 \mathrm{~min}$ at $4{ }^{\circ} \mathrm{C}$. The pellets were resuspended in tissue culture medium (DMEM 10\%) and B. besnoiti bradyzoites were counted in a Neubauer chamber in PBS containing 10\% trypan blue. Monolayers of Vero cells in $25 \mathrm{~cm}^{2}$ flasks were inoculated with $5 \times 10^{6}$ B. besnoiti bradyzoites. Infected cultures were passaged every 6 days.

At $48 \mathrm{~h}$ after inoculation, the medium was changed to DMEM 2\% FCSI with medium changes every 3 days. 
Infected cultures were inspected daily using an inverted microscope for the presence of free tachyzoites.

Once free tachyzoites were detected, cryopreservation of infected Vero cell cultures was done at the following passage, by resuspending infected Vero cells in FCSI containing 10\% DMSO, and freezing and storage in liquid nitrogen.

\subsection{Infection of rabbits with B. besnoiti bradyzoites and tachyzoites}

Two rabbits were inoculated intraperitoneally with $10^{7}$ B. besnoiti bradyzoites isolated from the Sallers' cysts, and were regularly inspected for the occurrence of skin lesions during 1 year. After 12 months, the same rabbits were inoculated with $10^{7}$ tachyzoites obtained from the respective in vitro culture, boosted with the same amount of tachyzoites 21 and 50 days later and were euthanized at 90 days post-inoculation. Serum and tissue specimens were collected for serology and histopathology, respectively. Animals were handled according to the legal stipulations of animal welfare.

\subsection{Indirect imunofluorescence antibody test} (IFAT) for the detection of anti-Besnoitia antibodies

Paraformaldehyde fixed $B$. besnoiti suspension $\left(2 \times 10^{6} \mathrm{ml}^{-1}\right)$ was obtained from cultured B. besnoiti tachyzoites from Israel (Kimron Veterinary Institute, Bet Dagan). Parasites were applied to microscopy slides in $6 \mu \mathrm{l}$, dried and fixed with cold acetone $\left(-20^{\circ} \mathrm{C}\right)$ for $10 \mathrm{~min}$, as described (Shkap et al., 2002). Serial two fold dilutions of serum samples in PBS were added and after $45 \mathrm{~min}$ at $37^{\circ} \mathrm{C}$, slides were washed with distilled water (three times, $10 \mathrm{~min}$ ) and droplets were covered with FITC conjugated rabbit anti-bovine IgG in PBS, incubated and washed as above. Bound antibodies were detected under $200 \times$ amplification using an UV light microscope Olympus BX50.

\subsection{Identification of B. besnoiti by PCR}

B. besnoiti infected Vero cell monolayers were scraped from the tissue culture flask using a rubber policeman. The parasites were separated from Vero cells and debris by passage through a Whatman CF-11 cellulose column as described (Shkap et al., 1984). Parasites were centrifuged at $770 \times g$ during $15 \mathrm{~min}$ at $4{ }^{\circ} \mathrm{C}$. The pellet was resuspended in PBS, and the tachyzoites were counted in a Neubauer chamber. A $10^{8}$ tachyzoites were centrifuged $\left(10,000 \times g\right.$ at $4{ }^{\circ} \mathrm{C}$ for $1 \mathrm{~min}$ ) and resuspended in $200 \mu \mathrm{lE}$, followed by the addition of $1 \mathrm{ml}$ of lysis buffer $(10 \mathrm{mM}$ Tris-Cl $(\mathrm{pH}$ 8.0); 0.1 M EDTA (pH 8.0); 0.5\% (w/v) SDS; $20 \mu \mathrm{g} / \mathrm{ml}$ RNase) to the cell suspension and a $1 \mathrm{~h}$ incubation at $37{ }^{\circ} \mathrm{C}$. For tissue digestion, $6 \mu \mathrm{l}$ of proteinase K $(20 \mathrm{mg} /$ $\mathrm{ml}$ ) were added to the lysate, mixed gently and incubated in a water bath for $3 \mathrm{~h}$ at $50{ }^{\circ} \mathrm{C}$, followed by phenol extraction as described by Sambrook and Russell (2001). DNA was solubilized in TE (pH 8.0) and stored over night at $4{ }^{\circ} \mathrm{C}$. DNA concentration was measured spectrophotometrically at $\lambda=260 \mathrm{~nm}$ (DU 68 Beckman Fullerton, USA).

Primers for polymerase chain reaction (PrCR) reaction were designed with primer $3^{\mathrm{TM}}$ software (Rozen and Skaletsky, 2000) using the published ITS1 partial DNA sequence of $B$. besnoiti $\left(G^{2}\right.$ Bank $^{\mathrm{TM}}$ accession number AF076859). The forward primer (5'-GGGTGCATTCGAGAAGTGTG-3') and reverse primer ( $5^{\prime}$-TCCGTGATAGCAGAGTGAGGAGG-3') were used for amplification of the B. besnoiti ITS1 sequence by PCR in an Eppendorf Mastercycler gradient Thermal Cycler (Hamburg, Germany), applying the following conditions: $3 \mathrm{~min}$ at $94{ }^{\circ} \mathrm{C}$ followed by 30 cycles of $30 \mathrm{~s}$ at $94{ }^{\circ} \mathrm{C}, 30 \mathrm{~s}$ at $65.5^{\circ} \mathrm{C}$, and $2 \mathrm{~min}$ at $72{ }^{\circ} \mathrm{C}$. A final extension of $5 \mathrm{~min}$ at $72{ }^{\circ} \mathrm{C}$ was used. PCR products were separated on $2 \%$ agarose gels and stained with $0.3 \mu \mathrm{g} / \mathrm{ml}$ ethidiumbromide.

\subsection{Transmission electron microscopy (TEM) and scanning electron microscopy (SEM)}

Both, small tissue pieces containing $B$. besnoiti cysts obtained from infected animals as well as $B$. besnoiti isolates (tachyzoites) were inspected by TEM. Samples were fixed in $2.5 \%$ glutaraldehyde in $100 \mathrm{mM}$ sodium cacodylate buffer ( $\mathrm{pH}$ 7.3) for $12-24 \mathrm{~h}$ at $4 \mathrm{C}$, were washed in cacodylate buffer and subsequently postfixed in $2 \% \mathrm{OsO} 4$ in cacodylate buffer for $4 \mathrm{~h}$ at room temp. Following extensive washing in water, specimens were left in $1 \%$ uranyle acetate for $1 \mathrm{~h}$, were washed in water, and were dehydrated in a graded series of ethanol. Specimens were embedded in Epon 812 resin, and sections were cut on a Reichert and Jung ultramicrotome. Sections were stained with uranyle acetate and lead citrate as described (Hemphill and Croft, 1997).

For SEM analysis, specimens were dehydrated by sequential incubations in increasing concentrations of ethanol (50-70\%), and were finally immersed in hexamethyl-disilazane and air-dried under a fume hood. They were then sputter-coated with gold, and inspected on a JEOL 840 scanning electron microscope operating at $25 \mathrm{kV}$. 


\section{Results}

As a prerequisite to this study, cattle in Portugal were surveyed for the occurrence of clinical signs of besnoitiosis. As there are other diseases with similar signs (burns; mange; fungus infection), it was necessary to confirm $B$. besnoiti infections serologically by detection of anti-B. besnoiti antibodies and by IFAT, and by direct histopathological detection of the parasite in the skin, isolation of tachyzoites in cell culture, and molecular confirmation of its identity by PCR. Confirmed clinical cases are reported from a large area in the south of Portugal (Alentejo).

In two animals presenting severe skin lesions reminiscent of B. besnoiti infection (Fig. 1A), indirect diagnosis of besnoitiosis was conducted by IFAT and revealed a high antibody titer $(>1024)$, while direct detection of the parasite tissue cysts by histophatology from skin biopsies (Fig. 1B) confirmed the diagnosis. TEM of skin biopsies showed, that parasites were surrounded by a massive, two-layered cyst wall (Fig. 1C). A large number $(>500)$ of parasites were found to be located within a parasitophorous vacuole that is delineated by an intracellular tissue cyst wall and the parasitophorous vacuole membrane, followed distally by an outer cyst wall (Fig. 1C). Closer inspection of parasites by TEM revealed typical features of bradyzoite stage parasites, including a nucleus located in the posterior region of the cell, and a large number of micronemes at the anterior part (Fig. 1D and E). Bradyzoites in the periphery (Fig. 1D), near the inner cyst wall, appeared to release small vesiculated structures, which were mostly found in the vicinity of the tissue cyst wall. These vesicles were absent in the matrix surrounding the parasites located in the interior region of the tissue cyst (Fig. 1E). Interior bradyzoites were embedded in a granular matrix that fills out the intercellular spaces. The molecular nature of this material is still unknown. Inoculation of isolated $B$. besnoiti from both animals into Vero cell culture (Fig. 2A and C) showed that bradyzoites were moving over, under and around the Vero cells monolayer during the 5 days subsequent to the inoculation. During this time, bradyzoites were motile and employed movements such as circular gliding, upright twirling and helical gliding (data not shown). After these initial 5 days, the bradyzoites were not visible anymore by phase contrast microscopy. However, the presence of the parasite was confirmed in in vitro cultures initiated from both animals by the amplification of the ITS1 partial sequence DNA of
$149 \mathrm{bp}$, which was absent in material obtained from uninfected control cultures (Fig. 2B). The amplified products revealed $100 \%$ identity with the DNA fragment reported by Ellis et al. (2000) $\left(\mathrm{GenBank}^{\mathrm{TM}}\right.$ accession number AF076859).

At 30 and 40 days post-infection characteristic forms of $B$. besnoiti tachyzoites of both the Sallers' and the Charolay's isolate were visible. Parasites formed small plaques, gradually destroyed the Vero cell layer, and large number of tachyzoites were released into the culture medium (Fig. 2A and C). Continuation of the culture on the same monolayer resulted in complete destruction of host cells due to continuous tachyzoite proliferation within the next 2-3 days. TEM (Fig. 2D and E) showed that these parasites proliferated within a parasitophorous vacuole, surrounded by a distinct parasitophorous vacuole membrane, and tachyzoites exhibited typical features of apicomplexan tachyzoite stage parasites of other species such as anterior conoid, micronemes, rhoptries and dense granules. In contrast to bradyzoites, the mitochondria were clearly visible, and tachyzoites were much more densely packed during their intracellular phase, thus a granular cyst matrix like in bradyzoites was not discernable. The two isolates were named Bb1Evora03 (from the 4 years old Sallers cow) and Bb2Evora03 (from the 6 years old Charolais bull). They were further passaged on Vero cells, and were cryopreserved in liquid nitrogen.

The two rabbits that had been initially inoculated initially with $B$. besnoiti bradyzoites isolated from the Sallers' cysts did not exhibit any clinical signs of disease during the following 12 months. Subsequent inoculation of cell culture-derived tachyzoites did also not result in any clinical manifestation of besnoitiosis. At necropsy, no lesions were found and no cysts were observed by histopathology. Both animals presented a high serum titer of $>2048$ in the IFAT test using slides sensitized with the Israel isolate of $B$. besnoiti (data not shown).

\section{Discussion}

This paper reports on the isolation and description of two new isolates (Bb1Evora03 and Bb2Evora03) of $B$. besnoiti, obtained from two naturally infected cattle from Alentejo, south of Portugal. Although at present we consider them as separate isolates, there is, at present, no evidence that these two isolates differ in any way with regard to morphological, structural or molecular features. Ongoing studies should clarify this point in the future. Only few isolates have been obtained so far in South Africa and Israel, thus our report on the 

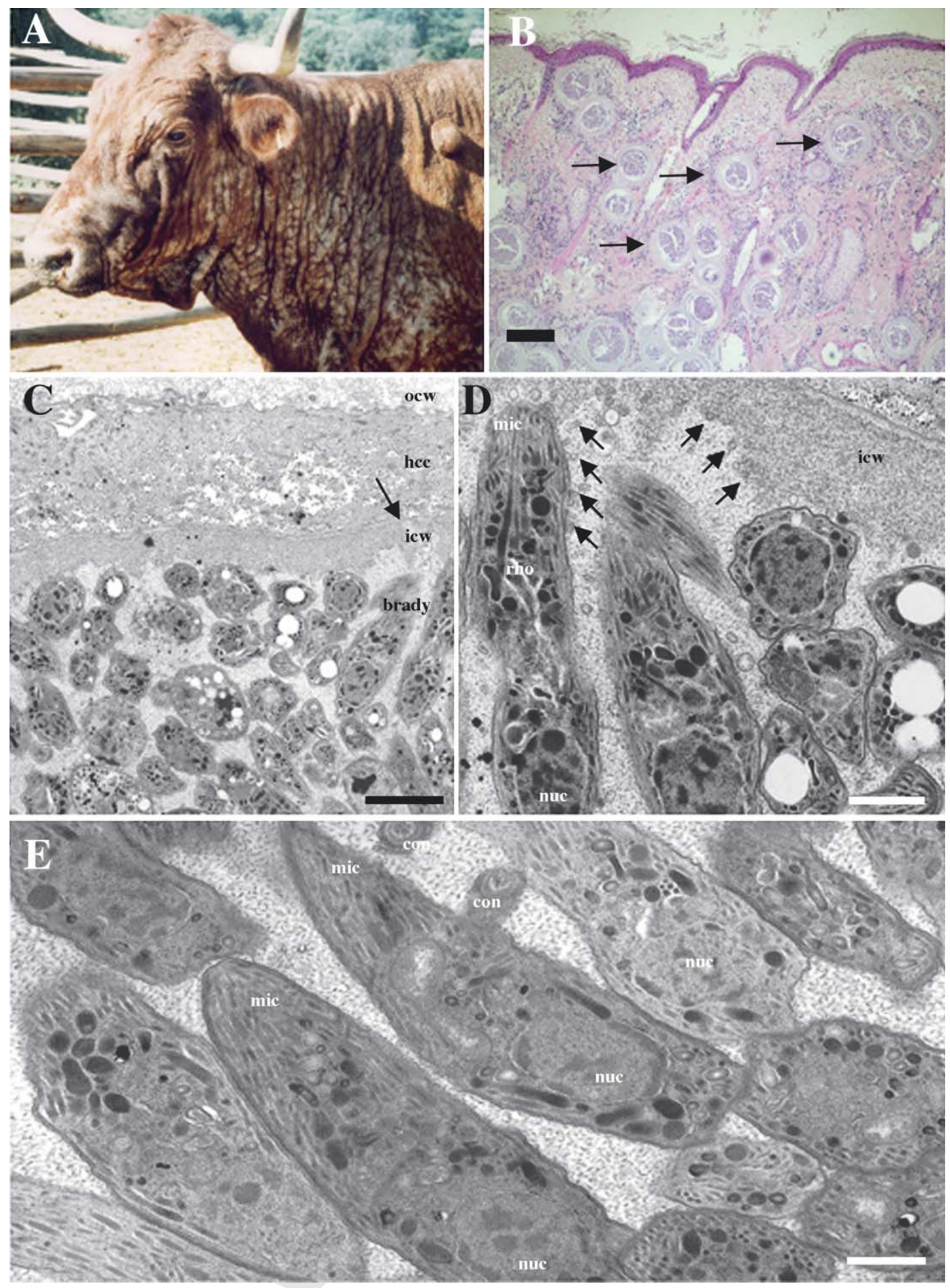

Fig. 1. Besnoitiosis in cattle. (A) A case of besnotiosis in a cow presenting severe besnoitiosis skin lesions. (B) Histopathology (paraffin section) of a skin lesion, stained with hematoxylin/eosin. The double-layered cysts are indicated with arrows. Bar $=200 \mu \mathrm{m}$. (C) TEM of Besnoitia cyst. Bradyzoites (brady) are located within an intracellular cyst, delineated by an intracellular cyst wall (icw) and the parasitophorous vacuole membrane (indicated by an arrow). Distally to the parasitophorous vacuole membrane, a portion of the host cell cytoplasm (hcc) and the outer cyst wall (ocw) is seen. Bar $=1.9 \mu \mathrm{m}$. (D) TEM of a Besnoitia cyst showing the peripheral region with parasites adjacent to the inner cyst wall (icw). Note presence of small vesiculated structures emanating from the parasites and incorporated into the cyst wall (arrows). nuc: nucleus, rho: rhoptries; bar $=0.9 \mu \mathrm{m}$. (E) TEM of the central portion of a Besnoitia cyst, showing numerous bradyzoites embedded in a granular matrix; nuc: nucleus; mic: micronemes; co: conoid; bar = $0.5 \mu \mathrm{m}$. 

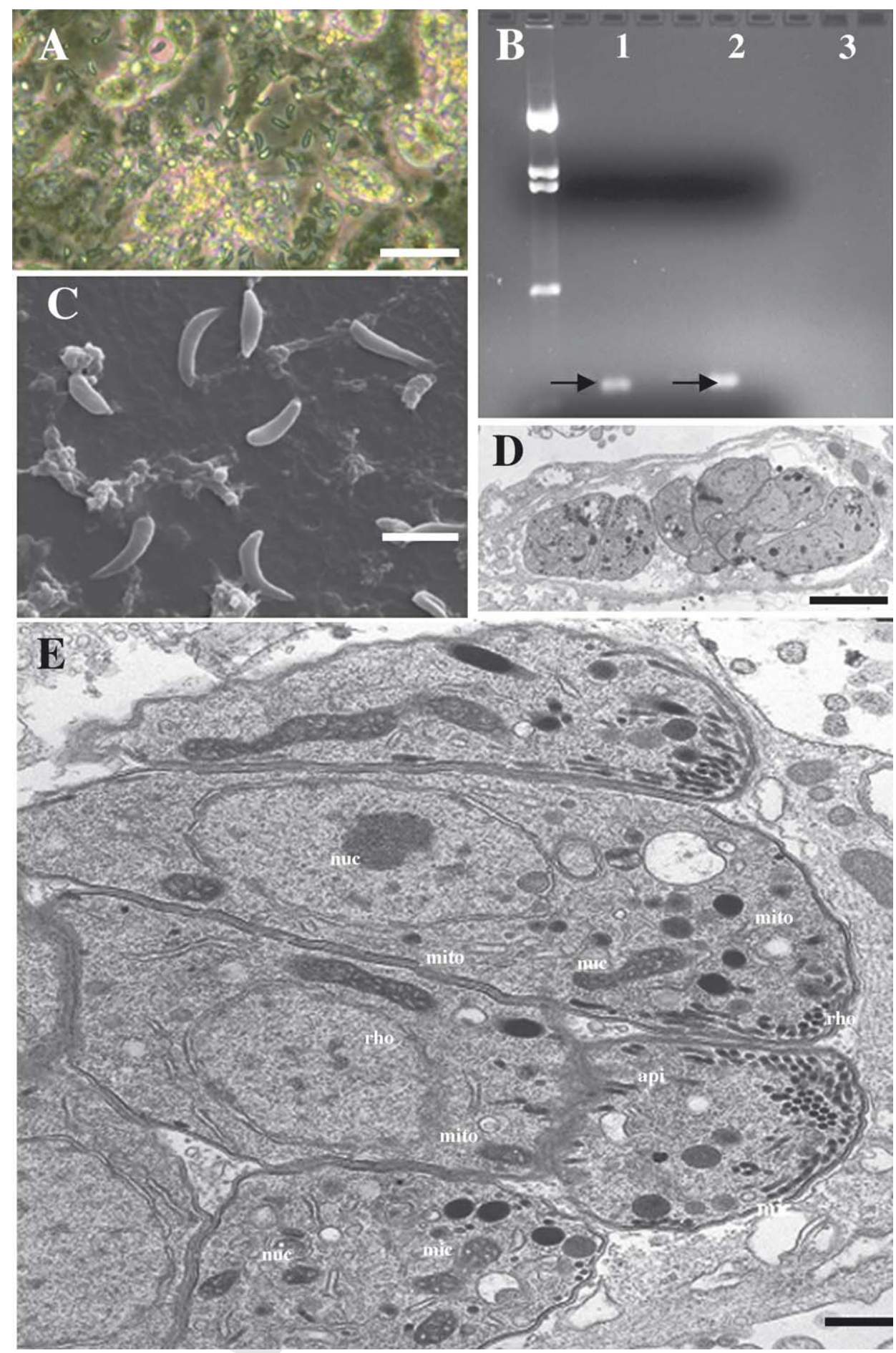

Fig. 2. In vitro culture of $B$. besnoiti. (A) Phase contrast micrograph of B. besnoiti in Vero cells, with tachyzoites emanating from host cells. (B) Molecular identification of $B$. besnoiti by PCR. ITS1 fragments of the isolates Bb1Evora03 (lane 1) and Bb2Evora03 (lane 2) by amplification of the 149 bp ITS1 fragments (arrows), while uninfected control cultures (lane 3) were negative. Molecular weight markers (MW) are pBR322 DNA BstN I digest Biolabs ${ }^{\circledR}$. (C) Scanning electron micrograph of Besnoitia tachyzoites in an infected Vero cell monolayer; bar $=12 \mu \mathrm{m}$. (D) TEM of infected Vero cell, showing tachyzoites situated within a parasitophorous vacuole; bar $=10 \mu \mathrm{m}$. (E) TEM of Besnoitia tachyzoites in Vero cell culture. Note that parasites are tightly packed, and consequently a cyst matrix is not visible. Nuc: nucleus; mito: mitochondria; mic: micronemes; api: apicoplast; bar $=0.3 \mu \mathrm{m}$. 
first European isolates may contribute to future studies on this barely known parasite with its increasing economical relevance.

While the $B$. besnoiti isolates obtained so far have been obtained by passage through laboratory animals, we are the first to describe the isolation of $B$. besnoiti directly by inoculation into cell culture. The fact that none of the two rabbits inoculated in this work showed any clinical signs of infection or lesions, as it was observed occasionally by others (Pols, 1960; Bigalke, 1968), reinforces the advantages of isolating these organisms directly in cell cultures. In the adaptation phase to in vitro conditions, meaning the first 30 days, the parasites were not identifiable by light microscopy, but clearly detectable by ITS1-based PCR. Thus, although there is a high geographical distance between our isolates (Bb1Evora03 and Bb2Evora03) from Portugal and those from South Africa on which the molecular phylogeny was published by Ellis et al. (2000), there is no difference in the available ITS1 sequences. This clearly suggests a high similarity between isolates from different geographical areas, and highlights the suitability of the primer pairs for future molecular confirmation of further $B$. besnoiti isolates.

The numbers of actual and new notifications of bovine besnoitiosis have grown in Europe (Cortes et al., 2003, 2004, 2005; Juste et al., 1990). Recently, Besnoitia tarandi has been isolated from reindeer in Finland (Dubey et al., 2004), and clinical besnoitiosis in roe deer (Capreolus capreolus) in Spain has been described (De Luco et al., 2000). These studies emphasize that infection by protozoans of the genus Besnoitia occurs in Europe more frequently than previously thought. The impact relative to sick animals at a farm level is related to $10 \%$ of the herd (Pols, 1960) and to a high number of infected animals, usually more than $80 \%$ (Bigalke, 1968).

Although our investigations are by no means comparable to an epidemiological study, we have demonstrated the presence of the disease in the south of Portugal, where beef production is the predominant output. In fact, in the majority of cases the disease had never been described in the farm, suggesting some dynamic of bovine besnoitiosis. Due to the overall skin lesions as shown in Fig. 1, and due to the secondary infections in wounds on areas of high elasticity demand, which cause a severe limitation to movement, animals end up in a severely impaired body condition. In addition, disease leads to painful breast feeding and increased abortion incidence in females and, in the males, to severe necrotizing orchitis and permanent infertility (Basson et al., 1970; Ferreira et al., 1982;
Cortes et al., 2005). In our field observations, during the initial phase of infection of a given herdaround $10 \%$ of animals die while in the acute stage of infection or in the chronic stage due to starvation. In addition, a large portion of animals are being culled due to the fact that they do not represent any commercial value anymore. After this dramatic, initial scenery on a herd, sporadic clinical cases, usually lower than $1 \%$, will occur. This just illustrates that basic biological questions regarding the life cycle, infection dynamics, and the host-parasite relationship more research on the infection biology of $B$. besnoiti is needed, and the isolation of this parasite will aid in those future investigations.

\section{Uncited references}

Frixione et al. (1996) and Sheffield (1968).

\section{Acknowledgements}

This work was supported by Centro de Investigação Interdisciplinar em Sanidade Animal (CIISA), Programa de Desenvolvimento Educativo para Portugal (PRODEP), and Instituto de Ciências Agrárias Mediterrânicas (ICAM). Andrew Hemphill was financially supported by the Swiss National Science Foundation (grant no. 3100AO-112532/1). We thank Professor Carlos Martins and through him the Laboratório de Doenças Infecciosas, Faculdade de Medicina Veterinária, Lisboa, Portugal for consistent support to our bench work and to Dr. Nuno Prates, Dr. José Miguel Leal da Costa, Dr. Miguel Fernandes and Dr. Vasco Fernandes for field sample collection and information on the occurrence of bovine besnoitiosis.

\section{References}

Basson, P.A., McCully, R.M., Bigalke, R.D., 1970. Observations on the pathogenesis of bovine and antelope strains of Besnoitia besnoiti, Marotel, 1912, infection in cattle and rabbits. Onderstepoort J. Vet. Res. 37, 105-126.

Besnoit, C., Robin, V., 1912. Sarcosporidioses cutanée chez une vache. Rec. Vet. 37, 649.

Bigalke, R.D., 1960. Preliminary observation on the mechanical transmission of cyst organisms of Besnoitia besnoiti, Marotel, 1912 from a chronically infected bull to rabbits by Glossina brevipalpis. Newstead, 1910. J. S. Afr. Vet. Assoc. 31, 37-44.

Bigalke, R.D., 1967. The artificial transmission of Besnoitia besnoiti, Marotel, 1912, from chronically infected to susceptible cattle and rabbits. Onderstepoort J. Vet. Res. 34, 303-316.

Bigalke, R.D., 1968. New concepts on the epidemiological features of bovine besnoitiosis as determined by laboratory and field investigations. Onderstepoort J. Vet. Res. 35, 3-138.

Bigalke, R.D., Schoeman, J.H., McCully, R.M., 1974. Immunization against bovine besnoitiosis with a live vaccine prepared from a 
blue wildebeest strain of Besnoitia besnoiti grown in cell cultures. 1. Studies on rabbits. Onderstepoort J. Vet. Res. 41, 1-5.

Bourdeau, P.J., Cesbron, N., Alexandre, F., Marchand, A.M., Desvaux, J.P., Douart, A., 2004. Outbreak of bovine besnoitiosis, Besnoitia besnoiti in the west of France and its diagnosis by immunofluorescence assay. In: Proceedings of the IX European Multicolloquium of Parasitology, Valencia, Spain, July 18-23, pp. 459-460.

Cortes, H., Ferreira, M.L., Silva, J.F., Vidal, R., Serra, P., Caeiro, V., 2003. Contribuição para o estudo da besnoitiose bovina em Portugal. Revista Portuguesa de Ciências Veterinárias 98 (545), 43-46.

Cortes, H., Leitão, A., Vidal, R., Reis, Y., Waap, H., Pereira da Fonseca, I., Fazendeiro, I., Ferreira, M.L., Caeiro, V., 2004. Bovine besnoitiosis, one approach for a better understanding of its importance in Portugal. In: Proceedings of the 23rd World Buiatrics Congress, Québec, Canada, July 11-16, pp. 35-36.

Cortes, H., Leitão, A., Vidal, R., Vila-Vicosa, M.J., Ferreira, M.L., Caeiro, V., Hjerpe, C.A., 2005. Besnoitiosis in bulls in Portugal. Vet Rec. 157, 262-264.

De Luco, F., Gortazar, C., Castillo, J., 2000. Besnoitiosis (Besnoitia sp.) in roe deer (Capreolus capreolus). In: Proceedings of the Fourth Meeting of the European Wildlife Disease Association. Zaragosa, Spain, p. 55

Dubey, J.P., Shkap, V., Pipano, E., Fish, L., Fritz, D.L., 2003. Ultrastructure of Besnoitia besnoiti tissue cysts and bradyzoites. J. Eukaryot. Microbiol. 50, 240-244.

Dubey, J.P., Sreekumar, C., Rosenthal, B.M., Vianna, M.C., Nylund, M., Nikander, S., Oksanen, A., 2004. Redescription of Besnoitia tarandi (Protozoa: Apicomplexa) from the reindeer (Rangifer tarandus). Int. J. Parasitol. 34, 1273-1287.

Ellis, J.T., Holmdahl, O.J., Ryce, C., Njenga, J.M., Harper, P.A., Morrison, D.A., 2000. Molecular phylogeny of Besnoitia and the genetic relationships among Besnoitia of cattle, wildebeest and goats. Protist 151, 329-336.

Elsheikha, H.M., Mansfield, L.S., 2004. Determination of the activity of sulfadiazine against Besnoitia darlingi tachyzoites in cultured cells. Parasitol. Res. 93, 423-426.

Ferreira, M.L., Nunes Petisca, J.L., Dias, O.H., 1982. Alterações testiculares em touros de Moçambique assintomáticos e com sintomas clínicos de besnoitiose. Rep. Trab. I. N. V. XIV 97-108.

Franco, E.E., Borges, I., 1915. Nota sobre a sarcosporidiose bovina. Revista de Medicina Veterinária Ano XIV, 255-299.

Frixione, E., Mondragon, R., Meza, I., 1996. Kinematic analysis of Toxoplasma gondii motility. Cell Motil. Cytoskeleton 34, 152 163.

Hemphill, A., Croft, S.L., 1997. Electron microscopy in parasitology. In: Rogan, M.T. (Ed.), Analytical Parasitology. Department of Biological Sciences, University of Salford, Salford, UK, pp. 227 268.
Janitschke, K., De Vos, A.J., Bigalke, R.D., 1984. Serodiagnosis of bovine besnoitiosis by ELISA and immunofluorescence tests. Onderstepoort J. Vet. Res. 51, 239-243.

Juste, R.A., Cuervo, L.A., Marco, J.C., Oregui, L.M., 1990. La besnoitiosis bovina: desconocida en España? Medicina Veterinária 7, 613-618.

Neuman, M., 1974. Cultivation of Besnoitia besnoiti Marotel, 1912, in cell culture. Tropenmed. Parasitol. 25, 243-249.

Pols, J.W., 1954. Preliminary notes on the behaviour of Globidium besnoiti, Marotel, 1912 in the rabbit. J. S. Afr. Vet. Med. Assoc. 25, 45-48.

Pols, J.W., 1960. Studies on bovine besnoitiosis with special reference to the aetiology. Onderstepoort J. Vet. Res. 28, 265-356.

Pipano, E., 1997. Vaccines against hemoparasitic diseases in Israel with special reference to quality assurance. Trop. Anim. Health Prod. 29, 86S-90S

Rozen, S., Skaletsky, H., 2000. Primer3 on the WWW for general users and for biologist programmers. In: Krawetz, M.S. (Ed.), Bioinformatics Methods and Protocols: Methods in Molecular Biology. Humana Press, Totowa, NJ, pp. 365-386.

Sambrook, J., Russell, D.W., 2001. Molecular Cloning. A Laboratory Manual, 3rd ed. Cold Spring Harbour Laboratory Press.

Sheffield, H.G., 1968. Observations on the fine structure of the "cyst stage" of Besnoitia jellisoni. J. Protozool. 15, 685-693.

Shkap, V., Ungar-Waron, H., Pipano, E., Greenblatt, C., 1984. Enzyme linked immunosorbent assay for detection of antibodies against Besnoitia besnoiti in cattle. Trop. Anim. Health Prod. 16, 233-238.

Shkap, V., Pipano, E., Greenblatt, C., 1987a. Cultivation of Besnoitia besnoiti and evaluation of susceptibility of laboratory animals to cultured parasites. Vet. Parasitol. 23, 169-178.

Shkap, V., Pipano, E., Ungar-Waron, H., 1987b. Besnoitia besnoiti: chemotherapeutic trials in vivo and in vitro. Rev. Elev. Med. Vet. Pays Trop. 40, 259-264.

Shkap, V., Yakobson, B.A., Pipano, E., 1988. Transmission and scanning electron microscopy of Besnoitia besnoiti. Int. J. Parasitol. 18, 761-766.

Shkap, V., Ungar-Waron, H., Pipano, E., 1989. Soluble antigens from culture-grown Besnoitia besnoiti endozoites. Vet. Parasitol. 34, $165-170$.

Shkap, V., Ungar-Waron, H., Pipano, E., 1990. Identification and partial purification of soluble antigens from culture-grown Besnoitia besnoiti endozoites. Rev. Elev. Med. Vet. Pays Trop. 43, 63 68.

Shkap, V., Pipano, E., 1993. The route of immunization of gerbils with live Besnoitia besnoiti as a factor in protection against lethal challenge. Vet. Parasitol. 51, 27-33.

Shkap, V., Reske, A., Pipano, E., Fish, L., Baszler, T., 2002. Immunological relationship between Neospora caninum and Besnoitia besnoiti. Vet. Parasitol. 106, 35-43. 